\title{
The effects of adrenergic and cholinergic agents upon eating and drinking in deprived rats
}

\author{
VINCENT P. HOUSER* \\ University of Massachusetts, Amherst, Mass. 01002
}

The effects of cholinergic and adrenergic agents on feeding and drinking behavior in deprived rats was investigated. Both scopolamine hydrobromide and scopolamine methylbromide reduced food intake, while decrements in water consumption were caused only by scopolamine hydrobromide. Dextroamphetamine sulfate reduced food intake but did not affect water consumption. Finally, both pilocarpine nitrate and DL-alpha-methyl-p-tyrosine had no effects on either measure. These results confirm Stein's (1963) suggestion that anticholinergic agents decrease eating via their peripheral effects while blocking drinking through their central activity. It was suggested that the motivational effects of these agents should be considered whenever these drugs are administered to animals that are under the control of appetitively motivated schedules of reinforcement.

Both scopolamine and atropine are known to reduce food intake in hungry rabbits and rats (Sadowski \& Longo, 1962; Pradham \& Roth, 1968). Specifically, it has been reported that scopolamine and atropine, along with their quarternary derivatives, decrease eating in motivated animals (Schmidt, Moak, \& Van Meter, 1958; Whitehouse, Lloyd, \& Fifer, 1964; Stein, 1963). Thus, it has been concluded that reduction in hunger is a peripheral effect of these anticholinergic agents (Stein, 1963). Thirst, on the other hand, is inhibited only by the central acting cholinergic agents (scopolamine and atropine). Therefore, it has been suggested that these agents block drinking via a central effect (Stein, 1963).

The available literature confirms that amphetamine is similar to the above anticholinergic agents in decreasing food and water intake in motivated animals. Miller (1956) has noted that racemic amphetamine sulfate in various doses leads to a decrease in food consumption in hungry rats. Other investigators have substantiated this finding (Cole, 1963). It has also been noted that amphetamine depresses thirst-induced water intake in the rat (Segal, Oden, \& Deadwyler, 1965; Morgenson, 1968).

Although evidence for the above agents is extensive, little is known about the motivational effects of drugs that are antagonistic to scopolamine and amphetamine. Specifically, it would be of interest to those who wish to examine the role of brain amines upon behavior maintained on appetitive schedules of reinforcement, whether cholinomimetic agents such as pilocarpine nitrate or

*The author wishes to express his appreciation to Miss Patricia Morrissey for drawing the figures and to Mrs. Frances L. Houser for typing the manuscript. adrenergic depleting agents such as alpha-methyl-p-tyrosine (alpha-MT) affect motivation.

\section{SUBJECTS}

The Ss for this experiment consisted of 45 male albino rats obtained from Gofmoor Farms, Westboro, Massachusetts. They ranged in age from 120 to 150 days at the beginning of the experiment.

\section{APPARATUS}

The experiment was conducted in the home cages of the animals. The food dishes used were circular, 4 in. in diam, and contained a pyramid-shaped cover with a $1 \frac{1}{2}$-in. hole cut in the center to give the animal access to the food inside the cup. This cover was used to force the animal to eat from the center of the cup so that any food dropped by the animal would remain in the cup's surface. The food used in these cups was Noyes precision rat food pellets $(4.0 \times 3.3 \mathrm{~mm}, 45 \mathrm{mg})$. Finally, $100-\mathrm{ml}$ drinking tubes were fastened to the home cages to monitor the amount of water consumed by each animal.

\section{PROCEDURE}

The 45 animals were randomly assigned to five separate drug groups. Each drug group was then divided into three separate dose conditions, each condition containing three animals.

The five drugs used were scopolamine hydrobromide, ${ }^{1}$ scopolamine methylbromide, pilocarpine nitrate, dextroamphetamine sulfate, ${ }^{2}$ and DL-alpha-methyl-p-tyrosine (alpha-MT). All the above drugs, with the exception of alpha-MT were dissolved in $.9 \%$ saline solution. Each drug was administered in the following doses: scopolamine hydrobromide and scopolamine methylbromide, $.1, .5$, and $1.0 \mathrm{mg} / \mathrm{kg}$ : pilocarpine nitrate, $1.0,2.5$. and $5.0 \mathrm{mg} / \mathrm{kg}$; and dextroamphetamine sulfate, $1.0, \quad 1.5$, and $3.0 \mathrm{mg} / \mathrm{kg}$.
Alpha-methyl-p-tyrosine was dissolved in a solution of $\mathrm{NaOH}$ and $\mathrm{HCl}$. This drug solution was made by adding $400 \mathrm{mg}$ of alpha-MT to $21.5 \mathrm{ml}$ of $1 \mathrm{~N} \mathrm{NaOH}$ and then adding $18.5 \mathrm{ml}$ of $1 \mathrm{~N} \mathrm{HCl}$. The final solution was then checked to determine that the $\mathrm{pH}$ was between 10.0 and 10.5 . The control solution, containing no drug, was made by simply mixing nearly identical amounts of $1 \mathrm{~N} \mathrm{HCl}$ and $\mathbb{N} \mathrm{NaOH}$ until the resulting $\mathrm{pH}$ was between 10.0 and 10.5 . The drug solution $(10 \mathrm{mg} / \mathrm{ml})$ of alpha-MT was administered in the three following doses: $25,50,75 \mathrm{mg} / \mathrm{kg}$. Unlike the other four drugs in which each dose was administered at a constant volume of $1 \mathrm{cc} / \mathrm{kg}$ of body weight, the alpha-MT dose groups were given a different volume of drug or control solution. Consequently, each dose group received a $10-\mathrm{mg} / \mathrm{ml}$ drug solution, but the higher dose levels received a larger volume of the solution. The $25-\mathrm{mg} / \mathrm{kg}$ dose group received a volume of $2.5 \mathrm{cc} / \mathrm{kg}$ of body weight, the $50-\mathrm{mg} / \mathrm{kg}$ group received $5.0 \mathrm{cc} / \mathrm{kg}$, while the $75-\mathrm{mg} / \mathrm{kg}$ group received $7.5 \mathrm{cc} / \mathrm{kg}$. The control solution was given in similar amounts. All injections were given intraperitoneally.

At the beginning of the experimental period all animals were brought down to $80 \%$ of their normal body weight within a 5-day period. The animals were then given 2 days in which to familiarize themselves with the food cups and the Noyes pellets. The cups were placed in the home cage for a 20-min session on each of these 2 days and the animals could eat as much as time allowed. The eating sessions occurred at the same time each day during the experiment. On the next 4 days all animals were given a saline injection $1 / 2 \mathrm{~h}$ before the 20-min eating session, with the exception of the alpha-MT groups. They were given the $\mathrm{NaOH} \mathrm{HCl}$ control solution $5 \mathrm{~h}$ before the session. After each session the food cups were weighed and compared to their weights before the session to determine how much food in grams had been consumed. The amount of water taken during the session was also recorded in milliliters from the drinking tubes attached to the home cages. The next 4 days were identical except that drug solutions were administered in the doses outlined above $1 / 2 \mathrm{~h}$ before the eating session. Doses of alpha-MT were given $5 \mathrm{~h}$ before the eating session because this drug produces its greatest physiological effect 5 to $7 \mathrm{~h}$ after its administration (Weissman \& Koe, 1965). After each daily session, the animals were given a sufficient supplement of Purina Lab Chow pellets to maintain a constant $80 \%$ body weight throughout the experiment. Finally, all animals had free 


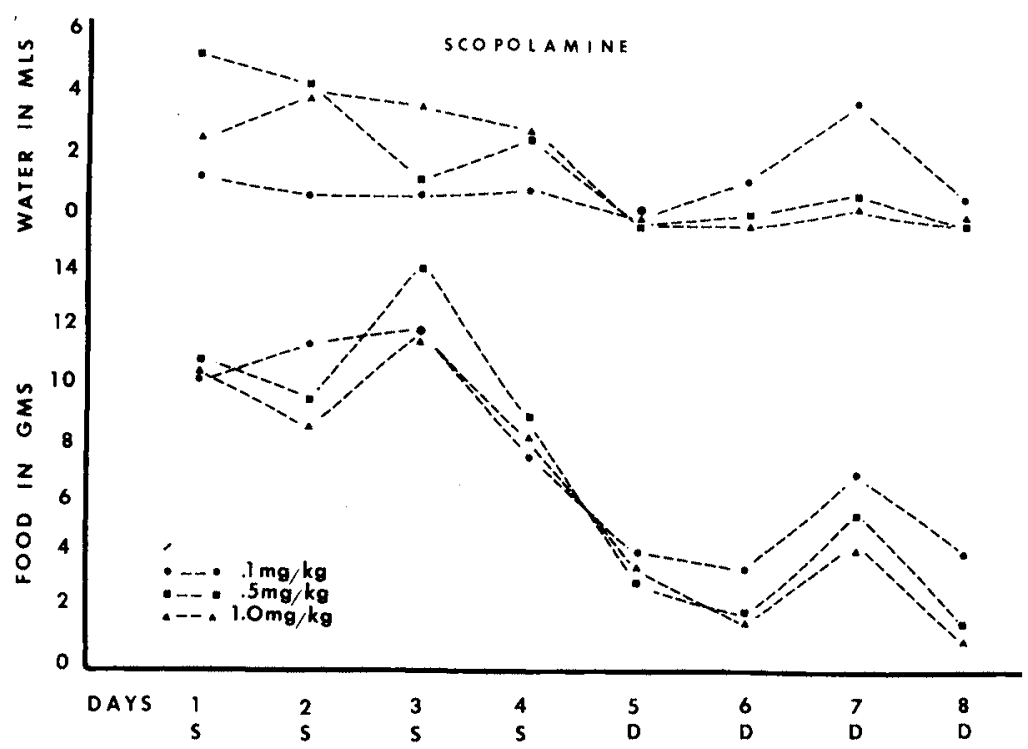

access to water during the entire experimental period.

\section{RESULTS}

Two separate analyses of variance were carried out on the data, one on each of the two measures. The analysis on food consumption indicated a significant overall drug effect $[F(4,30)=15.64, p<.01]$. Since there was no significant dose effect, it was possible to pool over dose levels and simply compare the control and drug conditions for each separate drug group in the subsequent $F$ tests. Figure 1 represents the plotted data for the scopolamine hydrobromide groups for amount of food and water consumed during the 8-day period.

A subsequent $F$ test indicated that the amount of food consumed under scopolamine hydrobromide was significantly less than that eaten under saline $[F(1,30)=251.43, \mathrm{p}<.001]$.

An analysis of the drinking data indicated a significant Dose by Condition interaction $[\mathrm{F}(2,30)=3.85, \quad \mathrm{p}<.005]$. This would suggest that some dose groups differed across drug saline conditions. Subsequent $F$ tests were carried out which showed that both the $.5-\mathrm{mg} / \mathrm{kg}$ $[F(1,30)=14.86, \quad p<.001]$ and the

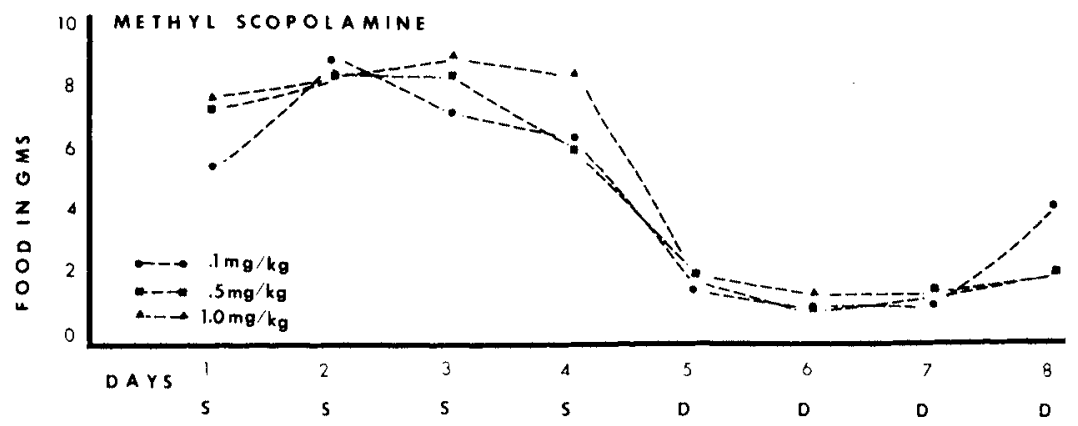

Fig. 1. Amount of food and water consumed by the three scopolamine hydrobromide dose groups during the 4 saline (S) and 4 drug (D) days.

In summary, only scopolamine hydrobromide, scopolamine methylbromide, and dextroamphetamine sulfate affected food consumption. In all cases, the doses used decreased the amount of pellets eaten. Furthermore, the higher doses did not differ significantly from the lower ones in their ability to depress food consumption. Water consumption was decreased by only the two higher doses of scopolamine hydrobromide.

\section{DISCUSSION}

The results of the present experiment are in agreement with the previous drug literature. Both scopolamine hydrobromide and its quaternary derivative decreased eating in motivated animals. Only the central acting agent, however, decreased water consumption during the daily sessions. This finding thus supports Stein (1963), who suggested that anticholinergic agents decreased food consumption in hungry animals via their peripheral effect while blocking drinking through their central activity. The detection of a decrease in drinking after drug administration was surprising considering the time water intake was monitored. Since the animals were hungry and not water deprived, the 20-min session was most likely to consist of food consumption behavior. Typically, animals spent most of the 20-min session eating and began drinking only when the food cups had been removed from the home cage. This would be expected, since animals were food deprived only and had constant access to water throughout the experimental period. The water measure was taken primarily to see if some of the drugs would decrease food intake by increasing drinking during the session. Thus, this measure was not taken over a long enough period to truly reflect changes in water consumption caused by drug administration. It was surprising then that this measure revealed the scopolamine effects and suggested that these decrements are probably quite severe and might have been detected even in the $.1-\mathrm{mg} / \mathrm{kg}$ group if the water measure had been taken over a longer period of time.

The amphetamine data were also in agreement with previous literature in that all three groups showed decrements in food consumption after drug administration.

Fig. 2. Amount of food consumed by the three scopolamine methylbromide dose groups during the 4 saline (S) and 4 drug (D) days. 


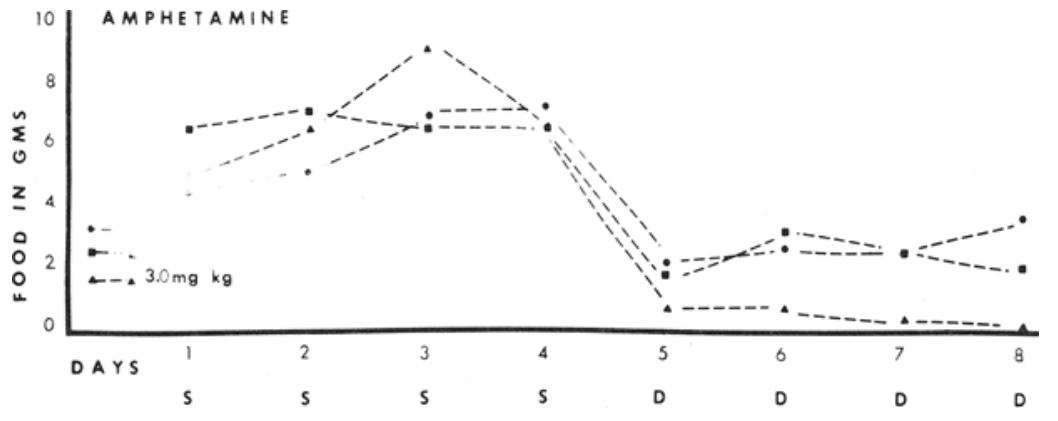

The lack of a decrement in drinking does not necessarily contradict the reports of several investigators (Segal et al, 1965; Morgenson, 1968) who noted such decreases after amphetamine administration. The insensitivity of the water measure used in this study may account for the above discrepancy.

Finally, both pilocarpine nitrate and alpha-MT do not seem to affect food consumption in hungry rats. Carlton \& Furgiuele (1965) have reported that alpha-methyl-m-tyrosine (alpha-MMT) does reduce consumption of sweetened milk in nondeprived rats. It should be remembered, however, that alpha-MMT depletes stores of serotonin as well as norepinephrine and dopa, while alpha-MT affects only the latter two brain amines (Hingtgen \& Aprison, 1963; Carlsson \& Lindquist, 1962. Furthermore, it has been suggested that alpha-MMT is transformed into "false transmitter" metabolites that replace and may functionally substitute for normal transmitter substances (Moore, 1966). In this regard, Carlton \& Furgiuele (1965) concluded that the anorexic effects of alpha-MMT were probably not due to the drug itself, but rather due to the metabolites of the agent. The fact that alpha-MT is not converted into "false transmitter" substances (Moore, 1966) may account for the differences between the Carlton and Furgiuele data and that reported in the present paper. Furthermore, the doses used in the present report $(25.75 \mathrm{mg} / \mathrm{kg})$ were much less than those employed by Carlton and Furgiuele who administered $100-400 \mathrm{mg} / \mathrm{kg}$ of alpha-MMT. Finally, the rats employed by the above authors were not deprived, while those in the present study were $80 \%$ of their normal free-feeding weights. These numerous differences between the experimental paradigms and drugs employed easily account for the discrepancy between the results of the present report and that of Carlton and Furgiuele.

In conclusion, it should be noted that whenever scopolamine or amphetamine are introduced in a food-reinforced operant task, the resulting changes in behavior may be due not only to the drug's effects on learning or retention, but also to their effects on motivation. Pilocarpine nitrate and alpha-MT, on the other hand, do not seem to affect appetitive motivation (at least in the doses employed in this study), so any behavioral changes noted after their administration cannot be ascribed to a change in motivation.

\section{REFERENCES}

CARLSSON, A.. \& LINDQUIST, M. In vivo decarboxylation of $\alpha$-methyl dopa and $\alpha$-methyl metatyrosine. Acta Physiologica Scandinavica. 1962.54. 87.94.

CARLTON. P. L.. \& FL:RGILELE, A. R. Appetite suppression due to $\alpha$-methyl-m-tyrosine. Life Sciences, 1964, 4. 1099-1106.

COLE, S. O. Interaction of amphetamine with conditions of food deprivation. Psychological Reports, 1963, 13, 387-390.
Fig. 3. Amount of food consumed by the three amphetamine sulfate dose groups during the 4 saline (S) and 4 drug (D) days.

HINGTGEN, J. N., \& APRISON, M. H. Behavioral response rates in pigeons: Effects of aipha-me thyl-m-tyrosine. Science, 1963, 141, 169-171.

VILLER. N. Effects of drugs on motivation: The value of using a variety of measures. Annals of the New York Academy of Sciences, 1956, 65, 318-333.

MOORE, K. E. Effects of alpha-methyltyrosine on brain catecholamines and conditioned behavior in guinea pigs. Life Sciences, 1966, 5 , 55-65.

MORGENSON, G. J. Effects of amphetamine on self stimulation and induced drinking. Physiology \& Behavior, 1968, 3, 133-136.

PRADHAM, S. N., \& ROTH, T. Comparative behavioral effects of several anticholinergic agents in rats. Psychopharmacologia, 1968, 12, 358-366.

SADOWSKI, B., \& LONGO, V. G. Electroencephalographic and behavioral correlates of an instrumental reward conditioned response in rabbits. A physiological and pharmacological study. Electroencephalography \& Clinical Neurophysiology, 1962, 14, 465-476.

SCHMIDT, V., MOAK, A., \& VAN METER, J. Atropine depression and water intake in the rat. American Journal of Physiology, 1958, $192,543-545$

SEGAL, E. F., ODEN, D., \& DEADWYLER, S. A. Determinants of polydipsia: V. Effect of amphetamine and pentobarbital. Psychonomic Science, 1965, 3, 33-34.

STEIN, L. Anticholinergic drugs and the central control of thirst. Science, $1963,139,46-48$.

WEISSMAN, A., \& KOE, B. K. Behavioral effects of L-alpha-methyltyrosine, an inhibitor of tyrosine hydroxylase. Life Sciences, 1965, 4, 1037-1048.

WHITEHOUSE, J. M., LLOYD, A., \& FIFER, A. Comparative effects of atropine and methyl atropine on maze acquisition and eating. Joumal of Comparative \& Physiological Psychology, 1964, 58, 475-476.

$$
\text { NOTES }
$$

1. Scopolamine hydrobromide and scopolamine methylbromide were kindly supplied by Sandoz Pharmaceuticals, Hanover, New Jersey.

2. Dextroamphetamine sulfate was kindly supplied by Smith, Kline and French Laboratories. Philadelphia, Pennsylvania. 